\title{
Erratum to: Intracranial germ cell tumors: a multi-institutional experience from three tertiary care centers in India
}

\author{
Aanchal Kakkar ${ }^{1} \cdot$ Ahitagni Biswas $^{2} \cdot$ Nikhil Kalyani $^{3} \cdot$ Uttara Chatterjee $^{4}$. \\ Vaishali Suri ${ }^{1} \cdot$ Mehar C. Sharma $^{1} \cdot$ Nishant Goyal $^{5} \cdot$ Bhawani S. Sharma $^{5}$. \\ Supriya Mallick ${ }^{2} \cdot$ Pramod K. Julka $^{2}$ - Girish Chinnaswamy ${ }^{3} \cdot$ Brijesh Arora $^{3}$. \\ Epari Sridhar $^{3} \cdot$ Sandip Chatterjee $^{6} \cdot$ Rakesh Jalali $^{3} \cdot$ Chitra Sarkar $^{1}$
}

Published online: 22 August 2016

(C) Springer-Verlag Berlin Heidelberg 2016

\section{Erratum to: Childs Nerv Syst}

DOI: 10.1007/s00381-016-3167-2

In the original publication of this paper, one of the author's names was spelled incorrectly. Instead of Ahitgani Biswas, it should be Ahitagni Biswas.

The online version of the original article can be found at http:10.1007 /s00381-016-3167-2.

\footnotetext{
Chitra Sarkar

sarkar.chitra@gmail.com

1 Department of Pathology, All India Institute of Medical Sciences, New Delhi 110029, India

2 Department Radiation Oncology, All India Institute of Medical Sciences, New Delhi 110029, India

3 Neuro Oncology Group, Tata Memorial Hospital, Mumbai 400012, India

4 Department of Pathology, IPGMER, Kolkata 700020, India

5 Department of Neurosurgery, All India Institute of Medical Sciences, New Delhi 110029, India

6 Department of Neurosurgery, Park Clinic, Kolkata, India
} 